\title{
Qualitative Assessment of the White Wine Varieties Grown in Dealu Bujorului Vineyard, Romania
}

\author{
Florin D. BORA ${ }^{1}$, Alina DONICI ${ }^{1}$, Aurel OşLOBANU², Avram FIȚIU ${ }^{3}$, \\ Anca C. BABEȘ ${ }^{4}$, Claudiu I. BUNEA ${ }^{4 *}$
}

\author{
${ }^{1}$ Research Station for Viticulture and Enology Târgu Bujor, Department of Agrochemistry, 805200 Târgu Bujor, \\ Romania; boraflorindumitru@gmail.com; donicialina79@gmail.com \\ 2"Iuliu Hațieganu" University of Medicine and Farmacy, Faculty of Medicine, Cluj-Napoca, Romania; oslobanu@yahoo.com \\ ${ }^{3}$ University of Agricultural Sciences and Veterinary Medicine, Faculty of Agriculture, 3-5 Mănăştur Street, 400372 Cluj-Napoca, \\ Romania;afitiu@yahoo.com \\ ${ }^{4}$ University of Agricultural Sciences and Veterinary Medicine, Faculty of Horticulture, Department of Horticulture and Landscaping, 3-5 \\ MănăşturStreet, 400372 Cluj-Napoca, Romania; babesanca@yahoo.com; claus_bunea@yahoo.com (*correspondingauthor)
}

\begin{abstract}
Ecoclimatic conditions have a great influence on grapevine growth and development. Favourable conditions (solar radiation, temperature, humidity etc.) are important and have a positive effect on the growth and fruition. Critical conditions have negative influences resulting in low grape production and wine quality. The purpose of this research was to analyse the quality of wine obtained from eight grapevine varieties (four Romanian autochthonous varieties: 'Fetească regală', 'Fetească albă', 'Băbească gri', 'Şarba', and four world-wide varieties: 'Aligoté', 'Sauvignon Blanc', 'Muscat Ottonel', and 'Italian Riesling'). The wine samples were obtained from micro-wine production under local weather conditions of Dealu Bujorului vineyard, Romania. The physico-chemical analysis of young wines showed that the highest alcohol content was recorded at the 'Sauvignon blanc' variety $(14.35 \%$ vol.) followed by 'Şarba' ( $14.10 \%$ vol.). The highest level of acidity was registered to 'Băbească gri' ( $\left.5.90 \mathrm{~g} / \mathrm{L} \mathrm{C}_{4} \mathrm{H}_{6} \mathrm{O}_{6}\right)$ and the lowest acidity in the 'Muscat Ottonel' wine $\left(4.40 \mathrm{~g} / \mathrm{L} \mathrm{C}_{4} \mathrm{H}_{6} \mathrm{O}_{6}\right)$. The $\mathrm{pH}$ values were between limits of 3.62 ('Fetească albă') and 3.27 ('Aligote'). In order to get a wider perspective about the wine quality, another 11 parameters were examined at these varieties, using spectrophotometric methods (acetic acid, potassium, calcium, free amino nitrogen, tartaric acid, copper, L-lactic acid, iron, L-malic acid, D-gluconic acid and glycerol). The results showed the suitability of ecoclimatic conditions and the proper growth and development of the tested varieties for obtaining wines with superiors quality.
\end{abstract}

Keywords: grapevine, Vitis vinifera, wine, quality, spectrophotometric method

\section{Introduction}

According to The International Organization of Vine and Wine (OIV) wine is a food product exclusively obtained by total or partial alcoholic fermentation of fresh grapes or the must obtained from pressed or unpressed grapes. From chemical point of view, wine is a complex beverage consisting of water, ethanol, sugar, amino acids, polyphenolic compounds, anthocyanins, organic and inorganic substances (Avram et al., 2014; Voica et al., 2009; Karataș et al., 2015).

Today the grapevine is cultivated all over the world, in both hemispheres of the Earth. Europe has the highest percentage $51 \%$ of the global surface cultivated with vines, followed by Asia, America, and Africa (Gonçalves da Silva et al., 2008). The wine-growing area in Romania has decreased since the 1990s, currently it ranks fifth in Europe after Spain, Italy, France and Portugal, so that in 2013 Romania had an area of 229000 hectares planted with vines (Bora et al., 2015).

The favourable ecoclimatic conditions for grapevine growth can be divided in two categories: vital conditions and critical ones. The vital conditions (solar radiation, temperature and humidity) are important and directly influence the growth and fruition of the grapevines. Critical conditions (frost, fog persistent, hoary frost, spring frosts) adversely affect growth and fruition of the grapevine, resulting in a decreased production both in terms of quantitative and qualitative values. (Webb et al., 2007; Pop, 2010; Camps and Ramos, 2011; Santos et al., 2012). Most of the authors argue that for determining the quality of a wine, some characteristics should be taken into 
594

account: the biochemical characteristics of the must and wine, the sugar content and its acidity (Bunea, 2010; Joshi et al., 2014; Yuyuen et al., 2015; Brunel et al., 2016; Catania et al., 2016). The grape quality is directly influenced by the variety, the ecoclimatic conditions, soil (physic-chemical characteristics of the soil), the wine-making process, the wine transportation and storage, the level of the agro-technical works applied and zoning (Coombe, 1987; Fernandez 1988; Núñez et al., 2000; Marini et al., 2006; Voica et al., 2009; Rotaru et al., 2010; Bora et al., 2015a; Condurso et al., 2015).

A favourable climate is essential for stabilizing the productivity, while the inter-annual variability of atmospheric conditions can strongly influence the quality of grapes (Jones and Goodrich, 2008) and consequently the quality of wines.

The Romanian wine industry is particularly involved in the controversial effects posed by climate changes, even if the overall effects of the climate change on the Romanian viticulture are uncertain, it is known that the productivity of grapevines is decreased because of the abiotic stresses (freezing temperatures, increasing soil salinity and accumulation of plant protection products in soil) (Păltineanu $e t$ al., 2007; 2009; Dragotă et al., 2011).

The effects were numerous and classified as direct and indirect effects according to the scientific literature (Marta $e t$ al., 2010). As climate change affects the onset and duration of each phenological phase, it also affects the grape production in terms of quantity and quality (Coll et al., 2011; Brunel et al., 2016; Ollat et al., 2016). In addition, climate change has a great impact on viticulture, altering the relationship between plants and pests, pathogens and weeds, reducing the time used by the farmers to solve these problems. Furthermore, the changes can also occur due to the long-term responses of farmers.

The effects of climate change on the wine production were presented in details in the scientific literature from various points of view: the importance of this industry in the agro-food economic world, the spread of viticulture in new areas that haven't been cultivated previously, various effects of global warming and weather fluctuations on cultivation processes (Schultz, 2000; Tate, 2001; Van Leeuwen et al., 2009; Fraga et al., 2012; Ollat et al., 2016; Lorenzo et al., 2016).

The French term "Terroir" defines geographical and environmental origin of the place where the grapes grown, also include other characteristics such as: soil composition (soil density and minerals), sunlight and climate (temperature and precipitations) (Laville, 1990). The mineral soil composition has an essential influence on grape quality and also on the organoleptic wine properties. Grape berries contain minerals like: $\mathrm{Ca}, \mathrm{Fe}, \mathrm{Na}$ and are very rich in potassium, essential macronutrient for grapevine and grape berry growth and development. Thus, potassium is the main cation in must and wine (aprox. $900 \mathrm{mg} / \mathrm{L}$; Blouin and Cruège, 2003).

The purpose of this study was therefore to evaluate the influence of ecoclimatic conditions of 2015 in Dealu Bujorului vineyard, Romania on the quality of the white wines, and the links between content of macronutrients $(\mathrm{K}, \mathrm{Ca}, \mathrm{Fe}$ etc.) and the main quality parameters of wine (alcohol, total acidity, $\mathrm{pH}$ etc.).

\section{Materials and Methods}

\section{Study area}

The study area of Dealu Bujorului vineyard is located at $45^{\circ} 52^{\prime} 10^{\prime \prime}$ North, $27^{\circ} 55^{\prime} 8^{\prime \prime}$ East, in the Galați county, Romania, at a distance of $56 \mathrm{~km}$ from Bârlad city and $55 \mathrm{~km}$ from Galați city. Although the region has a long tradition in culture of vine, vineyard Dealu Bujorului was developed with the establishment of Research and Development Station for Viticulture and Enology Bujoru (RDSVV Bujoru), from Târgu Bujoru city. In Dealu Bujorului vineyard predominant soil is levigated chernozem having a clayey sand texture with $\mathrm{pH}$ between 6.4 and 8.1. Although moisture deficit, natural conditions (ecoclimatic and ecopedological) offer viable ecosystem for the development of vineyard.

The study area is 10.98 ha it is consisting of grape varieties: 'Muscat Ottonel' (8,333 vines; 2.0 ha cultivated area); 'Fetească albă' (3,000 vines; 0.72 ha cultivated area); 'Băbească gri' (5,185 vines; 1.27 ha cultivated area); 'Aligoté' (6,600 vines; 1.60 ha cultivated area); 'Şarba' (1,979 vines; 0.48 ha cultivated area); 'Sauvignon Blanc' (15,350 vines; 3.07 ha cultivated area); 'Fetească regală' (4,867 vines; 1.18 ha cultivated area); 'Italian Riesling' (2,667 vines; 0.66 ha cultivated area). All the vines are planted at $2 \mathrm{~m}$ between rows and $1.2 \mathrm{~m}$ between vines, were pruned according to the Guyot system and were grown on espelier.

\section{Climatic data}

The weather data used in this research was recorded at the weather forecasting center and the Agro Expert system of RDSVV Bujoru. Based on this data, some important ecoclimatic indicators for the growth and fruition of the grapevine were determined as follow: Global thermal balance $\left(\sum \mathrm{t}^{\circ} \mathrm{g}\right)$ are the sum of all positive average daily temperature from active period; Active thermal balance $\left(\sum \mathrm{t}^{\circ} \mathrm{a}\right)$ are the sum of all daily mean temperature $\leq 10^{\circ} \mathrm{C}$; Beneficial thermal balance $\left(\sum \mathrm{t}^{\circ} \mathrm{u}\right)$ are the sum of all daily mean temperature above $10^{\circ} \mathrm{C}$; thermal coefficient $(\mathrm{Ct})$; amount of monthly and annual precipitation; amount of hours with sun $\left(\sum \mathrm{ir}\right)$ and real insolation coefficient $(\mathrm{Ci})$. Ct is given by the ratio of the overall balance $\left(\Sigma t^{\circ} g\right)$ and the number of days in the active period; $\mathrm{Ci}$ is given by the ratio between the hours with sun and the growing season days. $\mathrm{Cp}$ is given by the ratio between the rainfall of the growing season (mm) and the number of days of the growing season (Pop, 2010). In order to get a clearer image about how climatic factors influence the growth and fruition of the grapevines, some interactions of climate factors were calculated: the real heliothermal index $\left(\mathrm{HI}_{\mathrm{r}}\right)$, the hydrothermal coefficient $(\mathrm{CH})$, the bioclimatic vineyard index, annual aridity index Martonne ( $\left.\mathrm{I}_{\mathrm{ar}-\mathrm{DM}}\right)$ (Martonne 1926), the Huglin index (HI) (Huglin, 1978), oenoclimatic skills index $\left(\mathrm{IAO}_{c}\right)$ and cooling nights index $(\mathrm{CI})$.

The Huglin index $(\mathrm{HI})$ is calculated using formula:

$H \mathrm{I}=\Sigma(30$ September $/ 1$ April $) \times 1 / 2 \times\left[\left(\mathrm{T}_{\text {man }}-10\right)\right]+\left(\mathrm{T}_{\text {max }}-10\right) \times \mathrm{d}$

In the Northern Hemisphere in the above formula, $T=$ the mean air temperature $\left({ }^{\circ} \mathrm{C}\right), \quad T x=$ maximum air temperature $\left({ }^{\circ} \mathrm{C}\right), d=$ length of day coefficient, ranging from 1.02 to 1.06 between $40^{\circ}$ and $50^{\circ}$ of latitude. For Romania $d$ is 1.04 . 
Oenoclimatic skills index $\left(\mathrm{IAO}_{\mathrm{e}}\right)$ is used to determine the favourable climate of the region and also to determine the synthesis of anthocyanins in the grapes. It was calculated using:

$$
\mathrm{IAO}_{\mathrm{e}}=\mathrm{T}+\mathrm{I}-(\mathrm{P}-250)
$$

In the above formula: $\mathrm{T}=$ the amount of active temperature from 01.IV - 30.IX; I = amount of hours of insolation in the same period and $\mathrm{P}=$ the amount of precipitation in the same period.

The determination of the cool night index (CI) is done as given further (Tonietto, 1999): In the Northern Hemisphere: $\mathrm{CI}=$ minimum air temperature in the month of September (mean of minim), in ${ }^{\circ} \mathrm{C}$.

The last one is a night coolness variable which takes into account the mean minimum night temperature during the month when ripening usually occurs beyond the ripening period. The purpose of cool night index is to improve the assessment of the qualitative of wine-growing regions, notably in relation to secondary metabolites (aromas, polyphenols) in grape. That climatic factor is important as regards grape and wine colour and aromas (Kliewer and Torres, 1972; Kliewer 1973; Tomana et al., 1979).

\section{Sample collection and microvinification process}

The samples used in this experiment were obtained from the wines produced from the varieties 'Muscat Ottonel', 'Fetească regală, 'Băbească gri', 'Aligoté, 'Şarba', 'Sauvignon Blanc', 'Fetească albă' and 'Italian Riesling' under the conditions of 2015 year from Dealu Bujorului vineyard. The wine samples resulted from micro-wine production.

The grapes were harvested on September 25, 2015 at full maturity. Around $5 \mathrm{~kg}$ of grapes/cultivar were collected from 10 vines/replication. Three repetitions/cultivar were used, placed in randomized blocks. The grapes were collected from the top, middle and lower of each vine, grapes exposed to the sun, but also from shaded, thus obtaining a homogeneous samples. After sampling, the grape samples were placed in sealable plastic bag, and they were sent immediately to the laboratory for analysis. The samples of grapes were destemmed and crushed, then transferred to a microfermentor $(5 \mathrm{~L}$ cylindrical glass container, covered with aluminium foil to limit the effect of the light over the must) equipped with a fermentation airlock. Fermentation took place at $22-24^{\circ} \mathrm{C}$ and humidity $55-60 \%$. Afterwards wine was clarified by means of bentonite ( $40 \mathrm{~g} / \mathrm{L}$ 1:10 dilution) and combined with $\mathrm{SO}_{2}$ up to $100 \mathrm{~g} / \mathrm{L}$. Then wines were allowed to cool for thirty days at $-5^{\circ} \mathrm{C}$ for cold stabilization (Donnini et al., 2016). Then wine samples were stored in glass bottles at $5-6^{\circ} \mathrm{C}$ until the analyses.
Average data from three vinifications per cultivar are reported.

\section{Physical and chemical analysis}

The physical and chemical analysis of young wine were performed in the Winemaking Laboratory of the RSDVV Bujoru and were applied in accordance to the methods of analysis described in the Compendium of international methods of analysis of wines and musts (O.I.V., 2016), and to the Romanian STAS methods. During this analysis the following parameters were determined: alcohol (\% vol.) - was determined using the ebulliometric method, STAS 6182/6-70; total acidity $\left(\mathrm{g} / \mathrm{L} \mathrm{C}_{4} \mathrm{H}_{6} \mathrm{O}_{6}\right)$ - titrimetric method, STAS 6182-1:2008; volatile acidity $\left(\mathrm{g} / \mathrm{L} \mathrm{CH}_{3} \mathrm{COOH}\right)$ - according to STAS 61822:2008; free $\mathrm{SO}_{2}(\mathrm{mg} / \mathrm{L})$; total $\mathrm{SO}_{2}(\mathrm{mg} / \mathrm{L})$ - iodometric method, according to STAS 6182/13:2009; residual sugar $(\mathrm{mg} / \mathrm{L})$ - according STAS 6182/17-81 and the $\mathrm{pH}$ level was determined using WTW inoLab pH 7110. The next parameters: acetic acid $(\mathrm{g} / \mathrm{L})$; potassium $(\mathrm{mg} / \mathrm{L})$; calcium $(\mathrm{mg}$ $\mathrm{L})$; amino nitrogen $(\mathrm{mg} / \mathrm{L})$; tartaric acid $(\mathrm{g} / \mathrm{L})$; $\operatorname{copper}(\mathrm{mg} / \mathrm{L})$; 1-lactic acid $(\mathrm{g} / \mathrm{L})$; iron (mg/L); l-malic acid $(\mathrm{g} \mathrm{L}) ; \mathrm{D}$-gluconic acid $(\mathrm{g} / \mathrm{L}) ;$ glycerol $(\mathrm{g} / \mathrm{L})$ were determinate using spectrophotometric method.

\section{Reagents and general instrumental analysis}

All glassware and plastic-ware were soaked for at least $24 \mathrm{~h}$ in $10 \%(\mathrm{v} / \mathrm{v})$ nitric acid (Sigma-Aldrich) and then rinsed with copious amounts of water (18.2 M $\Omega$ ) (Millipore UV System). Solutions and dilutions were prepared using Grade 1 water.

In order to get a wider range of data about the quality of the tested wine and to determine the acetic acid, potassium, calcium, free amino nitrogen, tartaric acid, copper, L-lactic acid, iron, L-malic acid, D-gluconate and glycerol, the MIURA ONE I.S.E. S.r.l., Rome, Italy device was used. The operating parameters are shown in Table 1.

All reagents used for calibration were of analytical grade (TDI - Tecnología Difusión Ibérica, S.L. Fr.). Stock standard solutions were prepared daily or when an error is suspected to appear during preparation of the solutions. The intermediate solutions was stored in polyethylene bottles and glassware was cleaned by soaking in $10 \% \mathrm{v} / \mathrm{v} \mathrm{HNO}_{3}$ for 24 hours and rinsing at least ten times with ultrapure water. For quality control purpose, blanks and triplicates samples $(\mathrm{n}=3)$ were analyzed during the procedure. The variation coefficients were under $10 \%$ and detection limits $(\mathrm{mg} / \mathrm{L})$ were determined by the calibration curve method.

\section{Statistical analysis}

The statistical interpretation of the results was performed using the Duncan test, SPSS Version 23 (SPSS Inc., Chicago,

Table 1. Working parameters for MIURA ONE device

\begin{tabular}{|c|c|c|c|c|}
\hline Studied Parameter & Absorbance (nm) & Detection limit & Correlation coefficient & Dilution rate \\
\hline Acetic Acid & 340 & $0.03(\mathrm{~g} / \mathrm{L})$ & 0.9999 & $1: 1 ; 1: 2 ; 1: 5 ; 1: 30 ; 1: 1$ \\
\hline Potassium & 535 & $1.8(\mathrm{~g} / \mathrm{L})$ & 0.9999 & $1: 1 ; 1: 2 ; 1: 4 ; 1: 10 ; 1: 16 ; 1: 1$ \\
\hline Calcium & 578 & $0.6(\mathrm{mg} / \mathrm{L})$ & 0.9999 & $1: 1 ; 1: 2 ; 1: 4 ; 1: 10 ; 1: 1$ \\
\hline Amino Nitrogen & 340 & $3(\mathrm{mg} / \mathrm{L})$ & 0.9999 & $1: 1 ; 1: 2 ; 1: 4 ; 1: 10 ; 1: 1$ \\
\hline Tartaric Acid & 492 & $0.4(\mathrm{~g} / \mathrm{l})$ & 0.9999 & $1: 1 ; 1: 2 ; 1: 4 ; 1: 10 ; 1: 1$ \\
\hline Copper & 578 & $0.1(\mathrm{mg} / \mathrm{L})$ & 0.9999 & $1: 1 ; 1: 2 ; 1: 4 ; 1: 10 ; 1: 1$ \\
\hline L-Lactic Acid & 340 & $0.03(\mathrm{~g} / \mathrm{L})$ & 0.9999 & $1: 1 ; 1: 2 ; 1: 3 ; 1: 4 ; 1: 1$ \\
\hline Iron & 578 & $0.4(\mathrm{mg} / \mathrm{L})$ & 0.9999 & $1: 1 ; 1: 2 ; 1: 3 ; 1: 4 ; 1: 1$ \\
\hline L-Malic Acid & 340 & $0.03(\mathrm{~g} / \mathrm{L})$ & 0.9999 & $1: 1 ; 1: 2 ; 1: 4 ; 1: 7 ; 1: 1$ \\
\hline D-Gluconic Acid & 340 & $0.01(\mathrm{~g} / \mathrm{L})$ & 0.9999 & $1: 1 ; 1: 2 ; 1: 4 ; 1: 6 ; 1: 1$ \\
\hline Glycerol & 546 & $0.03(\mathrm{~g} / \mathrm{L})$ & 0.9999 & $1: 1 ; 1: 2 ; 1: 4 ; 1: 10 ; 1: 1$ \\
\hline
\end{tabular}


596

IL., USA). The statistical processing of the results was primarily performed in order to calculate the following statistical parameters: arithmetic average, standard deviation, standard error. This data was interpreted with the analysis of variance (ANOVA) and the average separation was performed with the DUNCAN test at $\mathrm{p} \leq 0.05$. In order to determine whether the major wine quality parameters may affect each other, the correlation coefficient was calculated using SPSS version 23 Pearson (SPSS Inc., Chicago, IL., USA).

\section{Results and Discussion}

\section{Study of ecoclimatic conditions}

The duration of the growing season is within its normal limits, over 170 days for the culture of grapevines (Pop, 2010), but in 2015 this limit was exceeded: were recorded 180 days for Dealul Bujorului Vineyard, Bujoru Wine Centre. Comparing these values (180 days) with the multiannual average (186 days) it can be observe a decrease of the vegetation period.

In the experimental year of 2015 the thermal balance values obtained are much lower than multiannual average: global thermal balance $\left(\Sigma \mathrm{t}^{\circ} \mathrm{g}\right)$ was $3469^{\circ} \mathrm{C}$ and active thermal balance $\left(\Sigma \mathrm{t}^{\circ} \mathrm{a}\right)$ was $3368^{\circ} \mathrm{C}$. In case of useful thermal balance multiannual average $\left(\Sigma \mathrm{t}^{\mathrm{o} u} 1667^{\circ} \mathrm{C}\right)$ was lower than 2015 $\left(\sum \mathrm{t}^{\circ} \mathrm{u} 1679^{\circ} \mathrm{C}\right)$. (Table 2).

Regarding the number of days with a maximum temperature of over $30^{\circ} \mathrm{C}$, in 2015 year was a 69 days, there is an increase comparing these values to multiannual average 43 days. The precipitation quantity in 2015 was lower (467.9 $\mathrm{mm})$ then average of the last ten years $(515.1 \mathrm{~mm})$. During the growing season, the recorded precipitations values were 218.2 $\mathrm{mm}$, below the multiannual average of $334.8 \mathrm{~mm}$ for Bujoru Wine Centre.

The insolation measured by the number of hours of sunshine and was higher than normal in the months during the growing season, with 1481 hours over the normal of 1431 hours (multiannual average). Insolation coefficient $\left(\mathrm{C}_{\mathrm{i}}\right)$ recorded value of 7.65 , this shows a decrease compared to the multiannual average (7.70).

In the climatic conditions of 2015, the real heliothermal index (HIr) value was 2.48 falling within the limits described in the scientific literature (1.35 and 2.70), which shows an increase in the heliothermal resources and optimal conditions for the ripening of late maturing variety (T,ârdea and Dejeu, 1995). Comparing with the multiannual average (2.40) it can be observed that in 2015 this parameter shows an increase. The hydrothermal coefficient $(\mathrm{CH})$ had a very low value of 0.65 , compared to the normal limits for our country, between 0.7 and 1.8, indicating that the humidity was insufficient, with recommendation for irrigation, for both table grapes and wine varieties. The viticultural bioclimatic index (Ibcv) with a value of 11.99 for 2015 shows that the heliothermal resources recorded high values due to low hydrous resources for the Bujoru Wine Centre (multiannual average was 7.9).

The oenoclimatic suitability index (IAOe) had a value of 4871 indicating an area with favourable conditions for growth of red grape varieties for wine, and also for the white wines. The Martonne aridity index had a value of 7.55 during the growing season, indicating a semiarid forest steppe climate. The heliothermal Huglin index provided useful information regarding the thermal potential for the culture of grape, both for table and wine grapes, with different periods of ripening. Compared to other heliothermal indices, it displays a close link with the sugar in the must. The sum of the Huglin index during the growing season was 2256 (multiannual average was 2251). The cooling nights index (CI) values were important especially during the ripening season. This value was obtained by summing up the minimum temperatures of the month. The CI index was calculated only for September and the obtained value was 12.9 , value that falls in the range of $12-14$ according to cool night climate class (multiannual average was 10.3).

The ecoclimatic conditions of Dealu Bujorului vineyard highlighted the exceptional viticultural characters of the Bujoru Wine Centre. These characters were found in the authenticity

Table 2. Ecoclimatic conditions in Dealu Bujorului

\begin{tabular}{|c|c|c|c|c|c|}
\hline & \multirow{3}{*}{ Climate conditions } & \multirow{3}{*}{$\frac{\text { Multiannual average }}{2005-2014}$} & \multicolumn{3}{|c|}{ Specific values } \\
\hline & & & \multirow{2}{*}{2015} & \multicolumn{2}{|c|}{ Extreme values } \\
\hline & & & & Min. & Max. \\
\hline The vegetation period & Days & 186 & 180 & 173 & 186 \\
\hline \multirow{7}{*}{ Thermal balance $\left({ }^{\circ} \mathrm{C}\right)$} & Global $\left(\sum \mathrm{t}^{\circ} \mathrm{g}\right)$ & 3469 & 3464 & 3125 & 3837 \\
\hline & $\operatorname{Active}\left(\sum \mathrm{t}^{\circ} \mathrm{a}\right)$ & 3368 & 3358 & 3201 & 3871 \\
\hline & Beneficial $\left(\sum \mathrm{t}^{\circ} \mathrm{u}\right)$ & 1667 & 1679 & 1432 & 1639 \\
\hline & Thermic Coefficient $\left(\mathrm{C}_{\mathrm{t}}\right)$ & 18.1 & 17.9 & 16.2 & 18.5 \\
\hline & Minimum absolute air temp. & -25.2 & -23 & -23.0 & - \\
\hline & Maximum absolute temp. & 41.5 & 37.3 & - & 37.3 \\
\hline & No. of days max temp. $>30$ & 43 & 69 & - & - \\
\hline \multirow{3}{*}{ Insolation (hours) } & Real $\left(\sum\right.$ ir $)$ & 1865 & 2025 & 1370 & 1678 \\
\hline & $\sum$ hours of insolation in the growing season & 1431 & 1481 & - & - \\
\hline & Insolation Coefficient $\left(\mathrm{C}_{\mathrm{i}}\right)$ & 7.70 & 7.65 & 6.66 & 8.57 \\
\hline \multirow{3}{*}{ Precipitations $(\mathrm{mm})$} & $\sum$ precipitations in the growing season & 334.8 & 218.2 & - & - \\
\hline & Annual $\left(\sum p p\right)$ & 515.1 & 467.9 & 431.8 & 529.1 \\
\hline & Precipitation Coefficient $(\mathrm{Cp})$ & 1.8 & 1.3 & 0.98 & 2.02 \\
\hline \multirow{7}{*}{$\begin{array}{l}\text { Interaction of climate } \\
\text { factors }\end{array}$} & Real Heliothermal index $\left(\mathrm{HI}_{\mathrm{r}}\right)$ & 2.40 & 2.48 & 2.06 & 2.34 \\
\hline & Hydrothermal coefficient $(\mathrm{CH})$ & 1.10 & 0.65 & 1.04 & 1.70 \\
\hline & Bioclimatic vineyard index (Ibcv) & 7.90 & 11.99 & 5.3 & 7.9 \\
\hline & Oenoclimatic skills index (IAOe) & 4680 & 4871 & - & - \\
\hline & Annual aridity index Martonne $\left(\mathrm{I}_{\mathrm{ar}-\mathrm{DM}}\right)$ & 26.3 & 23.6 & & \\
\hline & Heliothermal Huglin index $(\mathrm{HI})$ in the growing season & 2251 & 2256 & - & - \\
\hline & Cooling nights index $(\mathrm{CI})$ & 10.3 & 12.9 & - & - \\
\hline
\end{tabular}


and specificity of a wide assortment of wines obtained in the studied area. In this context it was expected that, in qualitative terms, the 8 varieties tested until now present a good adaptability and therefore the results of the physicochemical analysis indicate the production of quality wines.

\section{Analysis of the main quality parameters of wine}

Regarding the alcohol content of the tested wines, based on the results, we can state that the highest alcohol content was recorded at the 'Sauvignon Blanc' variety ( $14.35 \pm 0.25 \%$ vol.), followed by 'Sarba' and the lowest alcohol content was

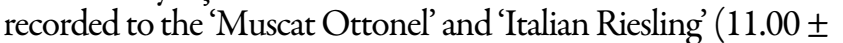

$0.29 \%$ vol.) varieties. It can also be seen that between the samples the differences were significant showing a values of $\mathrm{F}=$ $39.747, \mathrm{p} \leq 0.000$ (Table 3). The results are comparable with those reported by de Bruijn et al. (2014) (13.00 $\pm 0.0 \%$ vol. $13.60 \pm 0.10 \%$ vol.) for 'Sauvignon Blanc' wines from Chile and higher than those reported by Masneuf-Pomarède et al., 2006 for 'Sauvignon Blanc' in Bordeaux, France $12.7 \%$ vol.)

Total acidity belong the $\mathrm{pH}$ are of great importance for grape juice and wine stability, commonly used as indicators of quality. The wine must have a minimum content of $4.0 \mathrm{~g} / \mathrm{L}$ total acidity expressed as tartaric acid $(60$ millequivalent $/ \mathrm{L})$

Table 3. The analysis of the main wine quality parameters obtained in Dealul Bujorului Vineyard, 2015

\begin{tabular}{|c|c|c|c|c|c|c|c|c|c|}
\hline Variety & $\begin{array}{l}\text { Alcohol } \\
\text { (\% vol.) }\end{array}$ & $\begin{array}{c}\text { Total } \\
\text { Acidity } \\
(\mathrm{g} / \mathrm{L} \\
\left.\mathrm{C}_{4} \mathrm{H}_{6} \mathrm{O}_{6}\right)\end{array}$ & $\begin{array}{c}\text { Volatile } \\
\text { Acidity } \\
(\mathrm{g} / \mathrm{L} \\
\left.\mathrm{CH}_{3} \mathrm{COOH}\right)\end{array}$ & $\begin{array}{c}\text { Free } \mathrm{SO}_{2} \\
(\mathrm{mg} / \mathrm{L})\end{array}$ & $\begin{array}{c}\text { Total } \mathrm{SO}_{2} \\
(\mathrm{mg} / \mathrm{L})\end{array}$ & $\begin{array}{l}\text { Residual } \\
\text { sugar } \\
(\mathrm{mg} / \mathrm{L})\end{array}$ & $\begin{array}{l}\text { Non - } \\
\text { reducing } \\
\text { extract } \\
(\mathrm{g} / \mathrm{L})\end{array}$ & $\mathrm{pH}$ & $\begin{array}{l}\text { Acetic } \\
\text { Acid } \\
(\mathrm{g} / \mathrm{L})\end{array}$ \\
\hline 'Muscat & $11.00 \pm 0.88$ & $4.40 \pm 0.15$ & $0.54 \pm 0.04$ & $60.00 \pm 1$ & $240.00 \pm 6.4$ & $30.7 \pm 0.75$ & $29.00 \pm 0.50$ & $3.47 \pm 0.10$ & $0.143 \pm 0.004$ \\
\hline Ottonel' & $\mathrm{e}^{*}$ & e & b & $2 \mathrm{a}$ & $\mathrm{b}$ & $\mathrm{b}$ & b & b & $\mathrm{g}$ \\
\hline 'Fetească albă' & $\begin{array}{c}13.50 \pm 0.31 \\
\text { bcd }\end{array}$ & $\begin{array}{c}4.00 \pm 0.10 \\
\mathrm{f}\end{array}$ & $\begin{array}{c}0.39 \pm 0.03 \\
\text { c }\end{array}$ & $\begin{array}{c}6.40 \pm 0.2 \\
4 \mathrm{~g}\end{array}$ & $\begin{array}{c}89.60 \pm 1.86 \\
\mathrm{~g}\end{array}$ & nd & $\begin{array}{c}18.50 \pm 0.80 \\
\mathrm{f}\end{array}$ & $\begin{array}{c}3.62 \pm 0.01 \\
\mathrm{a}\end{array}$ & $\begin{array}{c}0.143 \pm 0.006 \\
\mathrm{~g}\end{array}$ \\
\hline 'Băbească gri' & $\begin{array}{c}13.20 \pm 0.16 \\
\mathrm{~cd}\end{array}$ & $\begin{array}{c}5.90 \pm 0.09 \\
\mathrm{a}\end{array}$ & $\begin{array}{c}0.38 \pm 0.02 \\
\text { c }\end{array}$ & $\begin{array}{c}50.00 \pm 2 \\
00 c\end{array}$ & $\begin{array}{c}250.40 \pm 2.0 \\
a\end{array}$ & $\begin{array}{c}12.70 \pm 0.3 \\
\mathrm{~d}\end{array}$ & $\begin{array}{c}23.50 \pm 1.00 \\
\mathrm{~d}\end{array}$ & $\begin{array}{c}3.53 \pm 0.03 \\
\mathrm{ab}\end{array}$ & $\begin{array}{c}0.317 \pm 0.012 \\
\mathrm{~d}\end{array}$ \\
\hline 'Aligoté' & $\begin{array}{c}13.10 \pm 0.08 \\
\mathrm{~d}\end{array}$ & $\begin{array}{c}5.50 \pm 0.09 \\
b\end{array}$ & $\begin{array}{c}0.37 \pm 0.04 \\
\mathrm{~b}\end{array}$ & $\begin{array}{c}48.60 \pm 0 \\
14 \mathrm{c}\end{array}$ & $\begin{array}{c}166.40 \pm 0.6 \\
3 \mathrm{e}\end{array}$ & nd & $\begin{array}{c}21.10 \pm 1.00 \\
\mathrm{e}\end{array}$ & $\begin{array}{c}3.27 \pm 0.03 \\
\mathrm{c}\end{array}$ & $\begin{array}{c}0.228 \pm 0.010 \\
\mathrm{e}\end{array}$ \\
\hline 'Şarba' & $\begin{array}{c}14.10 \pm 0.09 \\
\mathrm{ab}\end{array}$ & $\begin{array}{c}5.80 \pm 0.04 \\
\mathrm{a}\end{array}$ & $\begin{array}{c}0.54 \pm 0.02 \\
b\end{array}$ & $\begin{array}{c}52.00 \pm 0 . \\
6 \mathrm{~b}\end{array}$ & $\begin{array}{c}217.00 \pm 1.0 \\
\text { c }\end{array}$ & $\begin{array}{c}23.00 \pm 1.0 \\
0 c\end{array}$ & $\begin{array}{c}28.70 \pm 1.01 \\
c\end{array}$ & $\begin{array}{c}3.32 \pm 0.00 \\
\mathrm{c}\end{array}$ & $\begin{array}{c}0.339 \pm 0.029 \\
\text { c }\end{array}$ \\
\hline $\begin{array}{l}\text { 'Sauvignon } \\
\text { blanc' }\end{array}$ & $\begin{array}{c}14.35 \pm 0.25 \\
\mathrm{a}\end{array}$ & $\begin{array}{c}5.20 \pm 0.07 \\
\text { c }\end{array}$ & $0.57 \pm 0.04 \mathrm{ab}$ & $\begin{array}{c}20.0 \pm 0.6 \\
1 \mathrm{e}\end{array}$ & $\begin{array}{c}163.00 \pm 1.0 \\
\mathrm{e}\end{array}$ & $\begin{array}{c}12.00 \pm 0.5 \\
\mathrm{~d}\end{array}$ & $\begin{array}{c}27.00 \pm 1.00 \\
\mathrm{~b}\end{array}$ & $\begin{array}{c}3.54 \pm 0.17 \\
\mathrm{ab}\end{array}$ & $\begin{array}{c}0.478 \pm 0.009 \\
\mathrm{a}\end{array}$ \\
\hline $\begin{array}{l}\text { 'Fetească } \\
\text { regală' }\end{array}$ & $\begin{array}{c}13.80 \pm 0.20 \\
\text { bc }\end{array}$ & $\begin{array}{c}5.30 \pm 0.06 \\
c\end{array}$ & $0.42 \pm 0.04 c$ & $\begin{array}{c}38.4 \pm 0.5 \\
3 \mathrm{~d}\end{array}$ & $\begin{array}{c}140.8 \pm 1.77 \\
\mathrm{f}\end{array}$ & $\begin{array}{c}1.90 \pm 0.10 \\
\mathrm{e}\end{array}$ & $\begin{array}{c}20.00 \pm 1.00 \\
\text { ef }\end{array}$ & $\begin{array}{c}3.27 \pm 0.02 \\
c\end{array}$ & $\begin{array}{c}0.193 \pm 0.006 \\
f\end{array}$ \\
\hline $\begin{array}{l}\text { 'Italian } \\
\text { Riesling' }\end{array}$ & $\begin{array}{c}11.00 \pm 0.29 \\
\mathrm{e}\end{array}$ & $\begin{array}{c}4.90 \pm 0.05 \\
\mathrm{~d}\end{array}$ & $0.61 \pm 0.03 \mathrm{a}$ & $\begin{array}{c}18.0 \pm 0.3 \\
1 \mathrm{f}\end{array}$ & $\begin{array}{c}208.0 \pm 2.00 \\
\mathrm{~d}\end{array}$ & $\begin{array}{c}72.00 \pm 1.0 \\
\mathrm{a}\end{array}$ & $\begin{array}{c}38.00 \pm 1.00 \\
\mathrm{a}\end{array}$ & $\begin{array}{c}3.46 \pm 0.01 \\
b\end{array}$ & $\begin{array}{c}0.412 \pm 0.007 \\
b\end{array}$ \\
\hline Average & $13.04 \pm 0.28$ & $5.13 \pm 0.08$ & $0.48 \pm 0.03$ & $\begin{array}{c}36.68 \pm 0 \\
7\end{array}$ & $184.40 \pm 2.1$ & $19.04 \pm 0.5$ & $25.73 \pm 0.91$ & $3.43 \pm 0.04$ & $0.282 \pm 0.010$ \\
\hline $\begin{array}{l}\mathrm{F} \text { (Fisher } \\
\text { Factor) }\end{array}$ & 39.747 & 181.516 & 28.100 & 1365.189 & 1228.178 & 4285.033 & 140.311 & 10.556 & 340.214 \\
\hline Significance & $\mathrm{p} \leq 0.000$ & $\mathrm{p} \leq 0.000$ & $\mathrm{p} \leq 0.000$ & $\mathrm{p} \leq 0.000$ & $\mathrm{p} \leq 0.000$ & $\mathrm{p} \leq 0.000$ & $\mathrm{p} \leq 0.000$ & $\mathrm{p} \leq 0.000$ & $\mathrm{p} \leq 0.000$ \\
\hline Variety & $\begin{array}{l}\text { Potassium } \\
(\mathrm{mg} / \mathrm{L})\end{array}$ & $\begin{array}{l}\text { Calcium } \\
(\mathrm{mg} / \mathrm{L})\end{array}$ & $\begin{array}{l}\text { Amino- } \\
\text { Acid } \\
(\mathrm{mg} / \mathrm{L})\end{array}$ & $\begin{array}{c}\text { Tartaric } \\
\text { Acid } \\
(\mathrm{g} / \mathrm{L})\end{array}$ & $\begin{array}{c}\text { Copper } \\
(\mathrm{mg} / \mathrm{L}) / \\
\text { L-Lactic } \\
\text { Acid } \\
(\mathrm{g} / \mathrm{L})\end{array}$ & $\begin{array}{c}\text { Iron } \\
(\mathrm{mg} / \mathrm{L})\end{array}$ & $\begin{array}{c}\text { L-Malic } \\
\text { Acid } \\
(\mathrm{g} / \mathrm{L})\end{array}$ & $\begin{array}{c}\text { D-Gluconic } \\
\text { Acid } \\
\text { (g/L) }\end{array}$ & $\begin{array}{c}\text { Glycerol } \\
(\mathrm{g} / \mathrm{L})\end{array}$ \\
\hline $\begin{array}{l}\text { 'Muscat } \\
\text { Ottonel' }\end{array}$ & $\begin{array}{c}316.33 \pm 1.18 \\
h^{*}\end{array}$ & $93.49 \pm 1.67 \mathrm{a}$ & $\begin{array}{c}22.46 \pm 1.11 \\
\mathrm{a}\end{array}$ & $\begin{array}{c}1.69 \pm 0.06 \\
b\end{array}$ & ULD & $\begin{array}{c}1.64 \pm 0.10 \\
\mathrm{e}\end{array}$ & $\begin{array}{c}1.04 \pm 0.09 \\
\mathrm{~d}\end{array}$ & $\begin{array}{c}0.10 \pm 0.02 \\
\mathrm{~d}\end{array}$ & $\begin{array}{c}0.26 \pm 0.03 \\
c\end{array}$ \\
\hline 'Fetească albă' & $\begin{array}{c}645.96 \pm 9.79 \\
c\end{array}$ & $68.51 \pm 3.53 \mathrm{e}$ & $\begin{array}{c}6.59 \pm 0.14 \\
\mathrm{e}\end{array}$ & $\begin{array}{c}1.37 \pm 0.04 \\
\mathrm{~d}\end{array}$ & ULD & $\begin{array}{c}2.17 \pm 0.08 \\
\mathrm{~d}\end{array}$ & $\begin{array}{c}2.06 \pm 0.26 \\
\mathrm{ab}\end{array}$ & $\begin{array}{c}0.04 \pm 0.03 \\
\mathrm{e}\end{array}$ & $\begin{array}{c}0.28 \pm 0.03 \\
\text { bc }\end{array}$ \\
\hline 'Băbească gri' & $\begin{array}{c}494.60 \pm 4.99 \\
\mathrm{e}\end{array}$ & $75.69 \pm 2.23 \mathrm{~d}$ & $\begin{array}{c}2.52 \pm 0.13 \\
\mathrm{f}\end{array}$ & $\begin{array}{c}1.67 \pm 0.07 \\
b\end{array}$ & ULD & $\begin{array}{c}2.73 \pm 0.07 \\
b\end{array}$ & $\begin{array}{c}2.18 \pm 0.16 \\
a\end{array}$ & $\begin{array}{c}0.03 \pm 0.03 \\
\mathrm{e}\end{array}$ & $\begin{array}{c}0.21 \pm 0.02 \\
\mathrm{~d}\end{array}$ \\
\hline 'Aligoté' & $\begin{array}{c}349.93 \pm 6.37 \\
\mathrm{~g}\end{array}$ & $63.44 \pm 1.70 \mathrm{f}$ & $\begin{array}{c}22.86 \pm 1.01 \\
\mathrm{a}\end{array}$ & $\begin{array}{c}1.57 \pm 0.02 \\
\text { c }\end{array}$ & ULD & $\frac{1.51 \pm 0.03}{\mathrm{f}}$ & $\begin{array}{c}1.37 \pm 0.11 \\
\mathrm{c}\end{array}$ & $0.14 \pm 0.02 \mathrm{c}$ & $\begin{array}{c}0.30 \pm 0.05 \\
\mathrm{abc}\end{array}$ \\
\hline 'Şarba' & $\begin{array}{c}629.10 \pm 9.32 \\
\mathrm{~d}\end{array}$ & $88.63 \pm 1.34 b$ & $\begin{array}{c}21.59 \pm 0.99 \\
a\end{array}$ & $\begin{array}{c}1.75 \pm 0.09 \\
b\end{array}$ & ULD & $\begin{array}{c}2.29 \pm 0.03 \\
c\end{array}$ & $\begin{array}{c}1.91 \pm 0.04 \\
b\end{array}$ & $\begin{array}{c}0.20 \pm 0.02 \\
b\end{array}$ & $\begin{array}{c}0.29 \pm 0.02 \\
\text { bc }\end{array}$ \\
\hline $\begin{array}{l}\text { 'Sauvignon } \\
\text { blanc' }\end{array}$ & $\begin{array}{c}756.47 \pm 7.49 \\
b\end{array}$ & $67.47 \pm 1.34 \mathrm{ef}$ & $\begin{array}{c}13.58 \pm 0.83 \\
\text { c }\end{array}$ & $\begin{array}{c}1.09 \pm 0.03 \\
\mathrm{e}\end{array}$ & ULD & $\begin{array}{c}2.31 \pm 0.04 \\
\mathrm{c}\end{array}$ & $\begin{array}{c}1.43 \pm 0.21 \\
\mathrm{c}\end{array}$ & $\begin{array}{c}0.10 \pm 0.02 \\
\mathrm{~d}\end{array}$ & $\begin{array}{c}0.34 \pm 0.03 \\
\mathrm{ab}\end{array}$ \\
\hline $\begin{array}{l}\text { 'Fetească } \\
\text { regală' }\end{array}$ & $\begin{array}{c}370.80 \pm 8.64 \\
\mathrm{f}\end{array}$ & $83.64 \pm 3.22 c$ & $\begin{array}{c}14.64 \pm 1.15 \\
\text { bc }\end{array}$ & $\begin{array}{c}2.70 \pm 0.06 \\
\mathrm{a}\end{array}$ & ULD & $\begin{array}{c}3.07 \pm 0.04 \\
\mathrm{a}\end{array}$ & $\begin{array}{c}1.48 \pm 0.07 \\
\mathrm{c}\end{array}$ & nd & $\begin{array}{c}0.32 \pm 0.04 \\
\mathrm{ab}\end{array}$ \\
\hline $\begin{array}{c}\text { 'Italian } \\
\text { Riesling' }\end{array}$ & $\begin{array}{c}994.23 \pm 6.44 \\
\mathrm{a}\end{array}$ & $97.34 \pm 2.68 \mathrm{a}$ & $\begin{array}{c}16.10 \pm 0.77 \\
b\end{array}$ & $\begin{array}{c}1.42 \pm 0.04 \\
\mathrm{~d}\end{array}$ & ULD & $\begin{array}{c}1.67 \pm 0.08 \\
\mathrm{e}\end{array}$ & $\begin{array}{c}1.58 \pm 0.10 \\
\mathrm{c}\end{array}$ & $\begin{array}{c}0.26 \pm 0.03 \\
\mathrm{a}\end{array}$ & $\begin{array}{c}0.36 \pm 0.03 \\
a\end{array}$ \\
\hline Average & $569.68 \pm 6.78$ & $79.80 \pm 2.21$ & $15.04 \pm 0.77$ & $1.66 \pm 0.05$ & - & $2.17 \pm 0.06$ & $1.63 \pm 0.13$ & $0.11 \pm 0.02$ & $0.30 \pm 0.03$ \\
\hline $\begin{array}{l}\mathrm{F} \text { (Fisher } \\
\text { Factor) }\end{array}$ & 3106.332 & 89.523 & 227.831 & 237.177 & - & 230.165 & 20.376 & 48.991 & 6.943 \\
\hline Significance & $\mathrm{p} \leq 0.000$ & $\mathrm{p} \leq 0.000$ & $\mathrm{p} \leq 0.000$ & $\mathrm{p} \leq 0.000$ & - & $\mathrm{p} \leq 0.000$ & $\mathrm{p} \leq 0.000$ & $\mathrm{p} \leq 0.000$ & $\mathrm{p}=0.001$ \\
\hline
\end{tabular}

Average values, \pm standard deviation $(n=3)$.

*The difference between any two values, followed by a common letter is insignificant (Duncan test $\mathrm{p}<0.5$ )

$\mathrm{ULD}=$ under the limit of detection; nd= not detected 
598

(De La Hera Orts et al., 2005; Ţârdea, 2007; Yuyuen et al., 2015). The lack of acidity induces a flat taste in the wine and a weak storage endurance (mainly affected by the lactic acid bacteria and propionic). The highest level of acidity was registered in the 'Băbească gri' and 'Şarba' varieties which are equal in terms of statistics. In contrast to this, the lowest acidity level was recorded in the wines of 'Muscat Ottonel' and 'Fetească albă' varieties: $4.00 \pm 0.10\left(\mathrm{~g} / \mathrm{L} \mathrm{C}_{4} \mathrm{H}_{6} \mathrm{O}_{6}\right)$ (Table 3).

The volatile acidity sums up all the volatile fatty acids from the acetic series that can be found in wine in a free state or in the form of salts: acetic acid, formic, propionic, butyric, valeric, isovaleric and others. It represents about one tenth of the wine's acidity (Țârdea, 2007; Bhattacharjee, 2016). The wines from varieties 'Fetească albă: $\left(0.39 \pm 0.03 \mathrm{~g} / \mathrm{L} \quad \mathrm{CH}_{3} \mathrm{COOH}\right)$, 'Băbească gri' and 'Fetească regală' recorded the lowest level of volatile acidity compared with 'Italian Riesling: $0.61 \pm 0.03$ $\left(\mathrm{g} / \mathrm{L} \mathrm{CH}_{3} \mathrm{COOH}\right)$ with the highest volatile acidity (Table 3). The not fermented sugars in the wine (residual sugars) are present in small and variable amounts, usually between 2-80 $\mathrm{g} / \mathrm{L}$. Dry wines contain 2-3 g/L of sugars but do not compromise the wine's conservation/preservation. The amount of $2-5 \mathrm{~g} / \mathrm{L}$ sugar, give a smoother taste and an easier appreciable density, around 1,000 to the wine. More than $5 \mathrm{~g} / \mathrm{L}$ of sugar makes the wine sweet and fragile to microorganisms (Bhattacharjee, 2016). It can be observed that the analyzed varieties present significant differences $(\mathrm{F}=4285.033, \mathrm{p} \leq$ $0.000)$. The 'Italian Riesling' wine displayed the highest residual sugar content $(72.00 \pm 1.00 \mathrm{mg} / \mathrm{L})$, while the 'Fetească albă' and 'Băbească gri' wines had a sugar content below the detection limit of the analytical method. Our results were higher than those reported by Masneuf-Pomarède et al. (2006) for 'Sauvignon Blanc' in Bordeaux (0.7-1.7).

The dry extract refers to the assembly of all substances from wine or the substances that do not volatilize under wellestablished physical laboratory conditions. Such substances are found in a dissolved state or as colloidal suspensions, and their chemical nature is very different. The 'Italian Riesling' wine recorded the highest value of the irreducible extract $(38.00 \pm$ $1.00 \mathrm{~g} / \mathrm{L})$, followed by 'Muscat Ottonel' $(29.00 \pm 0.50 \mathrm{~g} / \mathrm{L})$. In contrary 'Fetească regală' had $20.00 \pm 1.00 \mathrm{~g} / \mathrm{L}$ and 'Fetească albă $18.50 \pm 0.80 \mathrm{~g} / \mathrm{L}$ (Table 3).

In contrast with the total acidity that expresses the titratable acidity of the must and wine, the $\mathrm{pH}$ represents a physicochemical index, which expresses the degree of ionization of the acid and their acid salts. In Dealu Bujorului vineyard 'Fetească albă' (3.62), 'Băbească gri' (3.53) and 'Sauvignon Blanc' (3.54) varieties had the highest $\mathrm{pH}$ values. Trigo-Córdoba et al. (2015) obtained comparable results for two white wines cultivated in Spain (3.22).

As it is formed in wine, the acetic acid hinders the activity of the yeasts fermentation and has the greatest contribution to the formation of the wine's volatile acidity, affecting, thus, the quality of the wine. Although a weak acidity has a great impact on wine taste; it imprints the taste of "vinegar" when its concentration exceeds 0.8-1.0 g/L (Bermeyer, 1987). The highest concentration of acetic acid was recorded in the 'Sauvignon Blanc' variety $(0.478 \mathrm{~g} / \mathrm{L})$ which was higher than those reported by Masneuf-Pomarède et al. (2006) for 'Sauvignon Blanc' cultivated in France (Bordeaux; max. 0.24 $\mathrm{g} / \mathrm{L})$.

Normally, wine contains $0.4-1.5$ g potassium per $\mathrm{L}$ (Țârdea, 2007), particularly in the form of KHT (bitartrate) which is stored. In a free state, the amount of potassium is lower; for example, the 'Chardonnay' wines from the Murfatlar Vineyard contain 410-496 K (mg/L), while the 'Sauvignon Blanc' 640$710 \mathrm{~K} \mathrm{mg} / \mathrm{L}$ (Marin et al., 1996); the red wines from UricaniIaşi contain $680-1125 \mathrm{~K} \mathrm{mg} / \mathrm{L}$ (Țârdea et al., 2001). The increasing concentration of potassium in wine occurs due to the use of chemical potassium fertilizers, irrigation and the addition of potassium metabisulfite to the wine that can reach up to 3.5-7.0 g of potassium bitartrate per Litre of wine (T,ârdea, 2007; Avram et al., 2014).

Based on the presented data it can be seen that potassium is found in high concentrations in wine. The highest concentration of $\mathrm{K}$ was recorded in the 'Italian Riesling' wine $(994.23 \pm 6.44 \mathrm{mg} / \mathrm{L})$. Contrasting examples are the wine from varieties of 'Aligote' $(349.93 \pm 6.37 \mathrm{mg} / \mathrm{L})$ and 'Muscat Ottonel' $(316.33 \pm 1.18 \mathrm{mg} / \mathrm{L})$. It can be claimed that the tested varieties of vines had a high influence on the accumulation of $\mathrm{K}$ in wine. The differences were statistically displayed $(\mathrm{F}=3106.332, \mathrm{p} \leq 0.000)$. The concentration of this element is in the range of normal limits compared to national and international data (Table 3). The results are similar to those reported by de Bruijn et al. (2014) (606.00 $\pm 7.00 \mathrm{mg} / \mathrm{L})$ for Chilean Sauvignon, and higher than those obtained in Romania by Avram et al. (2014) which reported average concentration $222.34 \pm 46.22 \mathrm{mg} / \mathrm{L}$ for 'Sauvignon Blanc', $235.24 \pm 46.34 \mathrm{mg} / \mathrm{L}$ for 'Fetească albă and average concentration $179.79 \pm 27.71 \mathrm{mg} / \mathrm{L}$ at 'Italian Riesling' variety.

Calcium is a natural component of must and wine. It accumulates in the grapes until ripening. The amounts are low, only 50-200 mg Cat $\mathrm{Ca}^{2+} \mathrm{L}$ of must. Wine always contains less calcium than must because of the alcohol content that contributes to the solidifications of calcium tartrate. White wines have a higher level in calcium than red wines and are prone to form tartaric deposits. For example, the red wines of Uricani-Iaşi have a calcium content of $56-88 \mathrm{mg} / \mathrm{L}$, and the white wines of Bucium-Iaşi 78-98 mg/L (Țârdea, 2007).

Regarding the calcium concentration of the wine, the 'Muscat Ottonel' (93.49 $\pm 1.97 \mathrm{mg} / \mathrm{L})$ and 'Italian Riesling' $(97.34 \pm 2.68 \mathrm{mg} / \mathrm{L})$ varieties reached the highest concentrations compared to the 'Aligote' $(63.44 \pm 1.70 \mathrm{mg} / \mathrm{L})$ variety which had the lowest concentration (Table 3). Avram et al. 2014 reported similar results $(62.54-77.36 \mathrm{mg} / \mathrm{L} \mathrm{Ca})$ for 'Sauvignon Blanc' variety cultivated in three different vineyards from Romania.

The evolution of assimilable nitrogen in the grapes is closely linked to the vine's metabolism. The variations between varieties are mostly determined by the content of the $\alpha$-amino nitrogen in the grapes and less by the ammoniacal nitrogen. During the ripening of grapes, the level of assimilable nitrogen increases progressively within relatively limited proportions: the $\alpha$-amino nitrogen content increases and the content of ammonical nitrogen decreases (Matthieu et al., 2001). Overall, the total assimilable nitrogen does not record a significant change. The decrease of the ammoniacal nitrogen in favour of the nitrogen amine corresponds to the nitrate metabolism of the grapes. Overripe grapes do not necessarily provide lower total assimilable nitrogen content, but a lower content of ammonia nitrogen. A content of amino nitrogen of $140 \mathrm{mg} / \mathrm{L}$ of must is estimated as limited value to the yeasts' activity (Gump et al., 2002; García-Ríos et al., 2014).

The tartaric acid is also known as "vinic acid" because is only formed in the green vine organs (vine and grapes). It is the most abundant and important acid in grapes and wines (60- 
$70 \%$ ) among the total acids. Starting from the must and up to the bottling of the wine, the content of tartaric acid is decreasing continuously. Thus, during the alcoholic fermentation, as the ethyl alcohol is being formed, about $50 \%$ of the must's tartaric acid has been deposited in the form of salts; the precipitation and deposition of potassium tartrate continues (KHT). Kept at cellar's temperature, white wines contain $6-30 \mathrm{mg} / \mathrm{L}$ of soluble tartrate and red wines $12-40$ mg/L (Pierre et al., 2005; Țârdea, 2007). The wine produced from the tested grapevine varieties had a low content of tartaric $\operatorname{acid}(\mathrm{g} / \mathrm{L})$ with value between $2.70 \mathrm{~g} / \mathrm{L}$ for 'Fetească regală' and $1.09 \mathrm{~g} / \mathrm{L}$ 'Sauvignon Blanc'. The results are lower than those reported by Masneuf-Pomarède et al. (2006) for 'Sauvignon Blanc' cultivated in Bordeaux area (5.30-6.61 g/L).

Due to mineral nutrition, grapevines accumulate small amounts of iron $2-3 \mathrm{mg} / \mathrm{L}$ in must. Further enrichment of must with exogenous iron is due to ground debris remaining on the grapes and due to the contact with bare metal parts of the wine machinery, reaching up to $20-30 \mathrm{mg} \mathrm{Fe} / \mathrm{L}$. Due to the reducing environment, during alcoholic fermentation some of the iron deposits and is removed from wine along with the yeast. As a result, the wine contains low amounts of iron, usually 4-5 mg/L (Olalla et al., 2000; Lara et al., 2005; Rousseva et al., 2013). Most of the iron in wine comes from storing the wine in bare metallic tanks and from the contact with wines conditioning machines (pumps, filters, hoses).

Regarding the iron content of wine (Table 3), the highest concentrations of $\mathrm{Fe}$ were recorded in the wines produced from varieties 'Fetească regală' $(3.07 \pm 0.04 \mathrm{mg} / \mathrm{L})$, and 'Băbească gri' and the lowest concentrations of Fe were recorded in 'Aligoté' variety $(1.51 \pm 0.03 \mathrm{mg} / \mathrm{L})$. Codreanu et al. (2014) recorded higher concentration of Fe (4.10-10.05 $\mathrm{mg} / \mathrm{L})$ in wine from 'Aligoté' variety cultivated in Bucium Vineyard, Romania.

The malic acid is the most common in nature, being synthesized by the green organs of the plants as a temporary measure between photosynthesis and cellular respiration. It represents about $70 \%$ of the total organic acids in the leaves. The high level of malic acid gave the organoleptic character of the "winy" undeveloped, harsh, acerbic taste. Only by reducing the malic acid concentration, the wine becomes more "rounded" and more enjoyable. The lowest concentration of Lmalic acid $(\mathrm{g} / \mathrm{L})$ was recorded in the wines from following varieties: 'Aligoté,, 'Sauvignon Blanc', 'Fetească regală' and 'Italian Riesling' which are equal in terms of statistics. The highest concentrations of L-malic acid were registered in wines produced from 'Băbească gri' $(2.18 \pm 0.16 \mathrm{~g} / \mathrm{L})$, followed by 'Fetească albă' $(2.06 \pm 0.26 \mathrm{~g} / \mathrm{L})$. The differences between varieties were statistically proved: $\mathrm{F}=20.376, \mathrm{p} \leq 0.000$ (Table 3).

The gluconic acid does not exceed $300 \mathrm{mg} / \mathrm{L}$ in must. In exchange, it is abundant in the wine from Aszú, Tokaj 0.5-2.5 g/L. During must's alcoholic fermentation, the gluconic acid does not undergo any changes (it is not metabolized by yeasts) and is entirely recovered in wine. It does not affect the wine quality. By EU regulation, the maximum content of gluconic acid allowed in wine is $1 \mathrm{~g} / \mathrm{L}$. Beyond this limit, the wines are suspected of gluconic acid addition.

The highest concentrations of d-gluconic acid $(\mathrm{g} / \mathrm{L})$ were registered in the 'Italian Riesling' wine $(0.26 \pm 0.03 \mathrm{~g} / \mathrm{L})$; in contrast, the lowest concentrations were recorded in 'Fetească albă' $(0.04 \pm 0.03 \mathrm{~g} / \mathrm{L})$ and 'Băbească gri' $(0.03 \pm 0.03 \mathrm{~g} / \mathrm{L})$, as shown in Table 3, which are similar in terms of statistics. The D-gluconic acid concentration in 'Fetească regală' was below the detection limit of the method and apparatus.

After water and alcohol, the glycerol is the most abundant in wine $5-15 \mathrm{~g} / \mathrm{L}$, depending on the health of the crop and the type of wine produced (dry or sweet). The wines from Tokaj Aszú are richer in glycerol, reaching up to $27 \mathrm{~g} / \mathrm{L}$. The large amount of glycerol formed during the fermentation process contributes to maintain the redox balance of the wine and to adjust the osmotic stress of the yeast in the case of sugar abundant musts (Zuzuarregui et al., 2005). The 'Italian Riesling' wine showed the highest concentration of glycerol $(0.39 \pm 0.03 \mathrm{~g} / \mathrm{L})$, followed by 'Fetească regală' $(0.32 \pm 0.04$ $\mathrm{g} / \mathrm{L})$, 'Sauvignon blanc' $(0.34 \pm 0.03 \mathrm{~g} / \mathrm{L})$ and 'Aligoté' $(0.30 \pm$ $0.02 \mathrm{~g} / \mathrm{L})$. The lowest concentration of glycerol was registered in the wine of 'Băbească gri' variety $(0.21 \pm 0.02 \mathrm{~g} / \mathrm{L})$. The difference between the varieties was statistically proved: $\mathrm{F}=$ 6.943, $\mathrm{p} \leq 0.000$ (Table 3).

In the case of $\mathrm{Cu}(\mathrm{mg} / \mathrm{L})$ and L-lactic acid $(\mathrm{g} / \mathrm{L})$, the concentration of these parameters was below the detection limit of the apparatus and the used method of analysis.

\section{The Pearson correlation between the main parameters analysed in wine}

In order to determine whether the main quality parameters of wine can influence each other, the Pearson correlation coefficient was calculated for each studied parameter as it shown in Table 4. A Pearson correlation coefficient value higher than 0.5 shows a strong correlation between the analysed varieties, a positive correlation between the two parameters shows that both parameters increased, a negative correlation indicates that a parameter increased while the second one decreased and vice-versa.

These provide a large number of both positive and negative correlations between the main parameters of the analysed wines. There are some relevant examples: Alcohol \& Sugar, $\left(r^{2}\right.$ $\left.=-0.697^{* *}\right)$; Volatile acidity \& Sugar, $\left(\mathrm{r}^{2}=-0.740^{* *}\right)$; Volatile acidity \& Dry Extract, $\left(\mathrm{r}^{2}=0.829^{* *}\right)$; Sugar \& D-gluconic Acid, $\left(r^{2}=0.733^{* *}\right)$; Dry Extract \& Calcium, $\left(r^{2}=0.722^{* *}\right)$; Dry Extract \& Iron, $\left(\mathrm{r}^{2}=-0.435^{*}\right)$; Dry Extract \& D-gluconic Acid, $\left(r^{2}=0.771^{* *}\right)$; $\mathrm{pH} \&$ Amine Nitrogen, $\left(\mathrm{r}^{2}=-0.571^{* *}\right)$; $\mathrm{pH} \&$ Tartaric Acid, $\left(\mathrm{r}^{2}=-0.583^{* *}\right)$, and other as it shown in Table 4. Opposite of mentioned above, in case of iron (F) and potassium $(\mathrm{P})\left(\mathrm{r}^{2}=-0.129\right)$, Paulette et al. (2015) reported the similar results for this correlation iron $(\mathrm{F})$ and potassium $(\mathrm{P})\left(\mathrm{r}^{2}\right.$ $=-0.090)$. In the case of Copper (C), L-lactic acid (Acil.), Total acidity (Acit.) and Glycerol (G) the values of the Pearson correlation coefficient for these parameters displayed no correlations.

Based on the previous Pearson correlation index, through this present research have been shown that the main parameters analysed from wine have had an influence on each other; in other words, the quality of the wine produced in the Vineyard of Dealu Bujorului is directly contingent on all these parameters.

\section{Conclusions}

The ecoclimatic conditions in the Dealul Bujorului, Bujoru Wine Centre, highlighted the exceptional viticultural value as well as the authenticity encountered in the wide variety of wines produced in the studied areas. Based on the results 
600

Table 4. Pearson correlation matrix between the main analysed wine parameters

\begin{tabular}{|c|c|c|c|c|c|c|c|c|c|c|c|c|c|c|}
\hline & Alc. & Aciv. & Sl. & St. & Sugar & ExtN. & $\mathrm{pH}$ & Acia. & P & $\mathrm{Ca}$ & Aami. & $\begin{array}{c}\text { Acid } \\
\text { tart. }\end{array}$ & $\mathrm{Fe}$ & $\begin{array}{c}\text { AciL } \\
\text { M. }\end{array}$ \\
\hline Alc. & 1.000 & & & & & & & & & & & & & \\
\hline Aciv. & -0.332 & 1.000 & & & & & & & & & & & & \\
\hline Sl. & -0.151 & -0.121 & 1.000 & & & & & & & & & & & \\
\hline St. & $-0.438^{*}$ & 0.340 & $0.697^{* *}$ & 1.000 & & & & & & & & & & \\
\hline Sugar & $-0.697^{* *}$ & $0.740^{* *}$ & -0.092 & $0.507^{*}$ & 1.000 & & & & & & & & & \\
\hline ExtN. & $-0.569^{* *}$ & $0.829^{* *}$ & -0.003 & $0.578^{* *}$ & $0.953^{* *}$ & 1.000 & & & & & & & & \\
\hline $\mathrm{pH}$ & -0.146 & 0.166 & $-0.490^{*}$ & -0.073 & 0.104 & 0.077 & 1.000 & & & & & & & \\
\hline Acia. & 0.211 & $0.539^{* *}$ & -0.244 & 0.280 & $0.429^{*}$ & $0.577^{* *}$ & 0.090 & 1.000 & & & & & & \\
\hline P & -0.060 & $0.583^{* *}$ & $-0.701^{* *}$ & -0.064 & $0.636^{* *}$ & $0.627^{* *}$ & 0.381 & $0.715^{* *}$ & 1.000 & & & & & \\
\hline $\mathrm{Ca}$ & $-0.600^{* *}$ & $0.604^{* *}$ & 0.249 & $0.535^{* *}$ & $0.774^{* *}$ & $0.722^{* *}$ & -0.133 & 0.037 & 0.213 & 1.000 & & & & \\
\hline Aami. & -0.225 & 0.392 & $0.449^{*}$ & 0.188 & 0.218 & 0.327 & $-0.571^{* *}$ & -0.068 & -0.215 & 0.315 & 1.000 & & & \\
\hline $\begin{array}{l}\text { Acid } \\
\text { tar. }\end{array}$ & 0.055 & -0.292 & 0.392 & -0.024 & -0.23 & 0.320 & $-0.583^{* *}$ & $-0.447^{*}$ & $-0.537^{* *}$ & 0.289 & 0.105 & 1.000 & & \\
\hline $\mathrm{Fe}$ & $0.592^{* *}$ & -0.289 & -0.038 & -0.150 & $-0.423^{*}$ & $-0.435^{*}$ & -0.045 & 0.039 & -0.129 & -0.101 & $-0.566^{* *}$ & $0.560^{* *}$ & 1.000 & \\
\hline $\begin{array}{l}\text { AciL- } \\
\text { M. }\end{array}$ & 0.347 & -0.359 & -0.258 & -0.091 & -0.166 & -0.251 & 0.266 & 0.107 & 0.273 & -0.225 & $-0.695^{* *}$ & -0.063 & $0.448^{*}$ & 1.000 \\
\hline $\begin{array}{l}\text { AciD- } \\
\text { G. }\end{array}$ & -0.374 & $0.619^{* *}$ & -0.015 & 0.339 & $0.733^{* *}$ & $0.771^{* *}$ & -0.200 & $0.489^{*}$ & $0.564^{* *}$ & $0.413^{*}$ & $0.544^{* *}$ & -0.380 & 0.039 & 0.107 \\
\hline
\end{tabular}

Alc. = alcohol (\% vol.); Aciv. = volatile acidity $(\mathrm{g} / \mathrm{L} \mathrm{CH} 3 \mathrm{COOH}) ; \mathrm{Sl} .=$ free sulphur dioxide $(\mathrm{mg} / \mathrm{L}) ; \mathrm{St} .=$ total sulphur dioxide $(\mathrm{mg} / \mathrm{L}) ;$ Sugar $=\operatorname{sugar}(\mathrm{mg} / \mathrm{L}) ; \mathrm{ExtN}$ $=\operatorname{dry} \operatorname{extract}(\mathrm{g} / \mathrm{L}) ;$ Acia. $=\operatorname{acetic} \operatorname{acid}(\mathrm{g} / \mathrm{L}) ; \mathrm{P}$. = potassium $(\mathrm{mg} / \mathrm{L}) ; \mathrm{Ca}$ = calcium $(\mathrm{mg} / \mathrm{L}) ; \operatorname{Aami} .=\operatorname{amino}$ nitrogen $(\mathrm{mg} / \mathrm{L}) ; \operatorname{Acid}$ tart. = tartaric acid $(\mathrm{g} / \mathrm{L}) ; \operatorname{AciL}-\mathrm{L}=$ L-lalic Acid (g/L); Fe = Iron (mg/L); AciL-M. = L-malic Acid (g/L); AciD-G. = D-gluconic acid (g/L);

the correlation is significant at $\mathrm{p}<0.05$ in $95 \% ;{ }^{* *}$ the correlation is highly significant at $\mathrm{p}<0.01$, in $99 \% ; \mathrm{N}=15$.

regarding the qualitative assessment of the tested varieties, they have a very good suitability in the studied areas. In terms of quality rating, they display particular characters of the varieties, as well as the ecoclimatic conditions and ecopedological influence on the quality of wine. The Pearson correlation index revealed the presence of a close relation between the main quality parameters of wine. Results also show that the wine produced from varieties cultivated in the Vineyard of Dealu Bujorului have a high content of macroelements ('Italian Riesling', 'Sauvignon blanc', 'Şarba'- K and 'Muscat Ottonel', 'Şarba', 'Fetească regală' - Ca), important for human's health. This higher content of some metals may be due to the viticultural practices, the use of fertilizers for cultivation $(\mathrm{K}, \mathrm{Ca}$, $\mathrm{Cu}$ ) the winemaking process or addition of substances for wine clearing as bentonite $(\mathrm{Na}, \mathrm{Ca}, \mathrm{Fe})$. Copper content is below the limit of detection due to the modern technology for obtaining wines in a controlled manner. The work offers new information on the quality of the white wines obtained in Dealu Bujorului, Romania, useful for their promotion and marketing.

\section{Acknowledgements}

This paper was published under the frame of the Romanian Ministry of Agriculture and Rural Development, project ADER no. 14.2.2. "Quantitative studies on assessment and monitoring contaminants, on the chain of viticulture and winemaking to minimize the amount of pesticides and heavy metals as principal pollutants" and by the Grant of the Romanian National Authority for Scientific Research and Innovation, CCCDI - UEFISCDI, project number 6/2015".

\section{References}

Avram V, Voica C, Hosu A, Cimpoiu C, Măruțoiu C (2014). ICP-MS characterization of some Romanian white wines by their mineral content. Revue Roumaine de Chimie 59(11-12):1009-1019.

Bora FD, Pop TI, Babeş AC, Popescu D, Iliescu M, Pop N (2015). Research on the Quality of the Three White Wines Varieties in Transylvania, Harvest of 2013-14. Bulletin of University of Agricultural Sciences and Veterinary Medicine Cluj-Napoca. Horticulture 72(2):327-334.
Bora FD, Bunea CI, Rusu T, Pop N (2015a). Vertical distribution and analysis of micro-, macroelements and heavy metals in the system soilgrapevine-wine in vineyard from North-West Romania. Chemistry Central Journal 9(1):19.

Bermeyer HD (1987). Methods of enzymatic analysis. Academic Press (2th ed), New York.

Bhattacharjee $S$ (2016). An analysis of classification techniques in data mining for determining quality of wine product: red \& white. Vidyaniketan Journal of Management and Research 4(1):161-177.

Blouin J, CruègeJ (2003) Analyse et Composition des Vins: Comprendrele Vin.[ Wine Analysis and Composition: Understanding Wine]. Editions La Vigne, Dunod, Paris, France, 304 pp.

Bunea CI (2010). Studies on variability of main productivity and quality characters in a collection of wine varieties concerning their suitability to organic culture technologies and marketing. $\mathrm{PhD}$ thesis, University of Agricultural Sciences and Veterinary Medicine Cluj-Napoca.

Bruijn J, Loyola C, Arumí L J, Martínez J (2014). Effect of non-protein factors on heat stability of Chilean Sauvignon Blanc wines. Chilean Journal of Agricultural Research 74(4):490-496.

Brunel A, Kernevez A, LeclereF, TrenteseauxJ (2016). Quantitative ranking evaluation of wine quality. International Journal of Biological, Biomolecular, Agricultural, Food and Biotechnological Engineering 10(2):78-84.

Catania P, De Pasquale C, Morello G, Vallone M (2016). Influence of grape transport and destemming systems on the quality of Chardonnay wines. Agricultural EngineeringInternational 18(2):260-266.

Camps JO, Ramos MC (2011). Grape harvest and yield responses to interannual changes in temperature and precipitation in an area of north-east Spain with a Mediterranean climate. International Journal of Biometeorology 56(5):853-864.

Codreanu M, Cotea VV, Luchian C, Niculaua M, Colibaba C (2014). Influence of pre-fermentative treatments on the composition of 'Tămâioaš românească and 'Aligoté' wines. Mitteilungen Klosterneuburg64(1):1-8.

Coombe BG (1987). Influence of temperature on composition and quality of grapes. Acta Horticulturae 206:23-35. 
Coll P, Le Cadre E, Blanchart E, Hinsinger P, Villenave C (2011). Organic viticulture and soil quality: A long term study in southern France. Applied Soil Ecology 50:37-44.

Condurso C, Cioncatta F, Tripodi G, Sparacio A, Giglio DMF, Sparla S, Verzera A (2015). Effects of cluster thinning on wine quality of Syrah cultivar (Vitis vinifera L.). European Food Research and Technology 246(10):1719-1726.

De La Hera Orts ML, Martínez-Cutillas A, López-Roca JM, Gómez-Plaza $\mathrm{E}$ (2005). Effect of moderate irrigation on grape composition during ripening. Spanish Journal of Agricultural Research 3(3):352-361.

Donnini S, Tessarin P, Ribera-Fonseca A, Di Foggia M, Parpinello G P, Rombolà AD (2016). Glyphosate impacts on polyphenolic composition in grapevine (Vitis vinifera L.) berries and wine. Food Chemistry 213:26-30.

Dragotă CS, Dumitrașcu M, Grigorescu I, Kucsicsa G (2011). The climatic water deficit in south Oltenia using the Thornthwaite method. Geography and Environmental Studies and Research 10(1):140-148.

Fernandez CP (1988). The importance of metallic elements in wine. A literature survey. Zeitschrift für Lebensmittel-Untersuchunf und Forschung 186(4):295-300.

Fraga H, Malheiro AC, Moutinho-PereiraJ, Santos JA (2012). An overview of climate change impacts on European viticulture. Food and Energy Security 1(2):94-112.

García-Ríos E, Gutiérrez A, Salvadó Z, Arroyo-López FN, Guillamona JM (2014). The fitness advantage of commercial wine yeasts in relation to the nitrogen concentration, temperature, and ethanol content under microvinification conditions. Applied and Environmental Microbiology 80(2)704713.

Gonçalves daSilva AM, Pavan MA, Muniz AS, Tonin TA, Pilizer T(2008). Nutrient availability in the soil and its absorption, transport, and redistribution in vines. Communications in Soil Science and Plant Analysis 39(9-10):1507-1516.

Gump BH, Zoecklein BW, Fugelsang KC, Whiton RS (2002). Comparison of analytical methods for prediction prefermentation nutritional status of grape juice. American Society for Enology and Viticulture 5(4):325-329.

Huglin P (1978). Nouveau mode d'évaluation des possibilities héliothermiques d'un milieu viticole. In: Proceedings of the Symposium International sur l'ecologie de la Vigne [New mode of evaluation of the solar thermal potential of an environment Viticulture. In: Proceedings of the International Symposium on Vine Ecology]. Contançapp 89-98.

Jones GV, Goodrich GB (2008). Influence of climate variability on wine regions in the western USA and on wine quality in the Napa Valley. Climate Research 35(3):241-254.

Joshi V, Kumar V, Debnath MK, Pattanashetti S, Variath MT, Khadakabhavi $S$ (2014). Assessing quality of blended wine prepared from white and red varieties of Grape (Vitis vinifera $\mathrm{L}$.). International Journal of Agricultural and Food Science 5(1):1-12.

Karataş D, Aydin F, Aydin I, Karataş H (2015). Elemental composition of red wines in Southeast Turkey. Czech Journal of Food Sciences 33(3):228-236.

Kliewer WM, Torres RE (1972). Effect of controlled day and night temperatures on grape coloration. American Journal of Enology and
Viticulture 2:71-77.

601

Kliewer WM (1973). Berry composition of Vitis vinifera cultivars as influenced by photo and nycto-temperatures during maturation. Journal of the American Society for Horticultural Science 2:153-159.

Lara R, Cerutti S, Salonia JA, Olsina RA, Martinez LD (2005). Trace element determination of Argentine wines using ETAAS and USNICP-OES. Food and Chemical Toxicology 43(2):293-297.

Laville P (1990) Le terroir, un concept indispensable a l'elaboration et á la protection des appellations d'origine comme a la gestation des vignobles: le cas de la France [Terroir, a concept indispensable to the elaboration and protection of appellations of origin as to the gestation of vineyards: the case of France].LeBulletin del'OIV709-710,217-241.

Lorenzo MN, Ramos AM, Brands S (2016). Present and future climate conditions for winegrowing in Spain. Regional Environmental Change 16(3):617-627.

Matthieu D, Dubernet M, Grosset F, Garcia A (2001). Analyse de l'azote assimilable dans les mouts par interférométrie infrarouge a transformé de Fourier, [Analysis of assimilable nitrogen in sewers by Fourier transform infrared interferometry]. Française d'œnologie 187:9-13.

Marin G, Lascău D, Mujdaba F (1996). Application film maceration before fermentation to obtain quality wines from Chardonnay and Sauvignon varieties in the Murfatlar vineyard. Annals ICVV 15:455-466.

Martonne E (1926). Une nouvelle fonction climatologique: L'indice d'aridité, [A new climatological function: The aridity index ]. La Météorologie 449-458.

Marta AD, Grifoni D, Mancini M, Storchi P, Zipoli G, Orlandini S (2010). Analysis of the relationships between climate variability and grapevine phenology in the Nobile di Montepulciano wine production area. The Journal of Agricultural Science 148(6):657-666.

Marini F, Bucci R, Magri AL, Magri AD (2006). Authentication of Italian $\mathrm{CDO}$ wines by class-modelling techniques. Chemometrics and Intelligent Laboratory Systems 84(1):164-171.

Masneuf-Pomarède I, Mansour C, Murat M-L, Tominaga T, Dubourdieu D (2006) Influence of fermentation temperature on volatile thiols concentrations in Sauvignon blanc wines. International Journal of Food Microbiology 108: 385-390.

Núñez M, Pena RM, Herrero C, García-Martín S (2000). Analysis of some metals in wine by means of capillary electrophoresis. Application to the differentiation of Ribeira Sacra Spanish red wine. Analusis 28(5):432437.

O.I.V. (2016). The International organization of wine and vine, 2016, Definitions and descriptions of the vitivinicultural products, Basic definition (18/73), I.3.1-1, Wines. Retrieved 2016 October 31 from http://www.oiv.int/en/technical-standards-and-documents/productsdefinition-and-labelling/definition-of-the-vitivinicultural-products-by code-sheet

Ollat N, Touzard JM, Leeuwen C (2016). Climate Change Impacts and Adaptations: New Challenges for the Wine Industry. Journal of Wine Economics 11(1):139-149.

Olalla M, González MC, Cabrera C, López AC (2000). Optimized Determination of Iron in Grape Juice, Wines, and Other Alcoholic Beverages by Atomic Absorption Spectrometry. Journal of AOAC International 83(1):189-195.

Paulette L, Man T, Weindorf DC, Person T (2015). Rapid assessment of 
602

soil and contaminant variability via portable $\mathrm{x}$-ray fluorescence spectroscopy: Copşa Mică, Romania. Geoderma 243-244:130-140.

Păltineanu C, Mihăilescu IF, Seceleanu I, Dragotă CS, Vasenciuc F (2007). Using aridity index to describe some climate and soil features in Eastern Europe: a Romanian case study. Theoretical and Applied Climatology 90(3):263-274.

Păltineanu C, Mihailescu IF, Prefac Z, Dragota CS, Vasenciuc F, Nicola C (2009). Combining the standardised precipitation index and climatic water deficit in characterising droughts: a case study in Romania. Theoretical and Applied Climatology 97:219-233.

Pop N (2010). Curs de viticultură generală [Course of general viticulture]. Eikon Press. Cluj-Napoca.

Pierre SB, Batlle LJ, Roset M (2005). Caractérisation de l'ajout d'acide métatartrique dans les jus de raisin, [Characterization of the addition of metatartaric acid in grape juice]. Bulletin OIV 78(897-898):779-801.

Rotaru L, Filipov F, Muste M, Soleru V (2010). Influence of some „Terroir Viticole" factors on quality of grape. Notulae Botanicae Horti AgrobotaniciCluj-Napoca38(1):176-181.

Rousseva M, Kontoudakis N, Schmidtke LM, Scollary GR, Clark AC (2016). Impact of wine production on the fractionation of copper and iron in Chardonnay wine: Implications for oxygen consumption. Food Chemistry 203:440-447.

Santos JA, Malheiro AC, Pinto JG, Jones GV (2012). Macroclimate and viticultural zoning in Europe: observed trends and atmospheric forcing. Climate Research 51:89-103.

Schultz HR (2000). Climate change in viticulture: A European perspective on climatology, carbon dioxide and UV effects. Australian Journal of Grape and Wine Research 6(1):2-12.

Tate AB (2001). Global Warming's Impact on Wine. Journal of Wine Research 12(2):95-109.

Tonietto J (1999). Les macrodimats viticoles mondiaux et linfluence du mésoclimat sur la typicité de la Syrah et du Muscat de Hambourg dans le sud de la France: méthodologie de caráctérisation, [Global wine macroclimates and the influence of mesoclimate on the typicity of Syrah and Muscat of Hamburg in the south of France: methodology of characterization]. $\mathrm{PhD}$ Thesis, Ecole Nationale Supérieure Agronomique, Montpellier.
Tomana T, Utsunomiya N, Dataoka I (1979). The effect of environmental temperatures on fruit on ripening on the tree. II. The effect of temperatures around whole vines and clusters on the coloration of 'Kyoho' grapes. Journal of the Japanese Society for Horticultural Science 48:261-266.

Trigo-Córdoba E, Bouzas-Cid Y, Orriols-Fernández I, Mirás-Avalos J M (2015). Effects of deficit irrigation on the performance of grapevine (Vitis vinifera L.) cv. 'Godello' and 'Treixadura' in Ribeiro. NW Spain. Agricultural Water Management 161:20-30.

T,ârdea C,Sârbu G, T,ârdea A (2001). Wine treated. "Ion Ionescu dela Brad" (Ed), Iasi, Romania.

Țârdea C (2007). Chemistry and wine analysis. "Ion Ionescu de la Brad" (Ed), Iasi, Romania.

Țârdea C, Dejeu L (1995). Viticulture. Didactic and Pedagogical RA (Ed), Bucureşti. Romania.

Voica C, Dehelean A, Pamula A (2009). Method validation for determination of heavy metals in wine and slightly beverages by ICPMS.Journal of Physics: ConferenceSeries 182(2009) 012036.

Van Leeuwen C, Tregoat O, Choné X, Bois B, Pernet D, Gaudillère JP (2009). Vine water status is a key factor in grape ripening and vintage quality for red Bordeaux wine. How can it be assessed for vineyard management purposes? Journal International des Sciences de la Vigne et duVin 43(3):121-134.

Webb LB, Whetton PH, Barlow EWR (2007). Modelled impact of future climate change on the 559 phenology of wine grapes in Australia. Australian Journal ofGrape and Wine Research 13:165-175.

Zuzuarregui A, Carrasco P, Palacios A, Julien A, del Olmo M (2005) Analysis of the expression of some stress induced genes in several commercial wine yeast strains at the beginning of vinification. Journal of Applied Microbiology 98:299-307.

Yuyuen P, Boonkerd N, Wanapu C (2015). Effect of grape berry quality on wine quality. Suranaree Journal of Science and Technology 22(4):349356. 